Research Article

\title{
A retrospective pilot study analyzing the effects of various factors and benefits of various drugs used in treating late admitted cases of acute ischemic stroke
}

\author{
Sharanabasayyaswamy B. Hiremath*, Sudhindra P. A.
}

Department of Pharmacology, SDM Medical College,

Dharwad, Karnataka, India

Received: 03 February 2016

Accepted: 02 March 2016

\section{*Correspondence to:}

Dr. Sharanabasayyaswamy B.

Hiremath,

Email: dr.sharan83@yahoo.com

Copyright: () the author(s), publisher and licensee Medip Academy. This is an openaccess article distributed under the terms of the Creative Commons Attribution NonCommercial License, which permits unrestricted noncommercial use, distribution, and reproduction in any medium, provided the original work is properly cited.

\begin{abstract}
Background: In Indian scenario, most of the cases of acute ischemic stroke are admitted and initiated treatment when the stipulated period of 4-6h for thrombolytic therapy is crossed. It is unclear on to benefits of treating such patients and our study aims at analysing the clinical and demographic factors associated with achieving improvement in functional ability in this special category of patients.
\end{abstract}

Methods: Review of case records of acute ischemic stroke patients admitted after $6 \mathrm{~h}$ of onset of stroke to a tertiary care teaching hospital of north Karnataka, India, over a period of one year with minimum of seven days of treatment was done. Drug utilization pattern, severity of ischemic stroke, baseline demographic and clinical data were extracted. The neurological recovery at the end of treatment period was analysed by using Barthel Index (BI) score. All the factors and drug utilization pattern were described using descriptive statistics and their correlation with BI score was analysed by spearman's correlation coefficient.

Results: Of the total 446 case records with diagnosis of stroke, only 39 met the eligibility criteria and included in the study. Statistically significant correlation to BI score was seen with severity of stroke, day-1 SBP, use of physiotherapy, time lapsed in initiating treatment, amount of decrease in SBP and baseline serum creatinine level.

Conclusions: Our study helped in generating the hypothesis on effects of various factors and therapeutic modalities on neurological recovery of late admitted cases of acute ischemic stroke.

Keywords: Retrospective pilot study, Acute ischemic stroke, Neurological deficit

\section{INTRODUCTION}

Stroke is defined as loss of brain function due to a disturbance in the blood supply to the brain either due to ischemia or hemorrhage. ${ }^{1}$ Stroke is one of the major cause of mortality and morbidity in India with its incidence on rise every decade. ${ }^{2}$ Four major classes of drugs along with thrombolytic agent used in treatment of acute stroke treatment includes anti-hypertensives, anticonvulsants, osmotic agents and neuroprotective agents. ${ }^{3}$ Thrombolytic agents are a major part of treatment in ischemic stroke and t-PA is the only drug proven to be effective and approved by FDA for acute ischemic stroke. ${ }^{4}$ Role of various other drugs used, especially the anti-hypertensive drugs in treating acute ischemic stroke is still unclear and controversial. ${ }^{5}$ In-fact a recently conducted meta-analysis opines that early reduction of blood pressure to normal level is of no benefit. ${ }^{6}$ Another interesting class of drugs used in prophylaxis and treatment of acute stroke are nootropic or neuroprotective agents. ${ }^{7}$ Though there are few such agents like citicoline and piracetam, most of them have failed to show major beneficial effects and benefits of their use is controversial. ${ }^{8,9}$ The role of anti-platelet drugs like low dose aspirin and clopidogrel in prophylaxis is though well proved, their benefits observed are moderate..$^{10,11}$ 
The outcome of treating a case of acute ischemic stroke is strongly dependent on timely medical intervention with a thrombolytic agent within 4-6 h of onset of stroke to lower the incidence of mortality and severity of morbidity. ${ }^{12}$ In Indian scenario it would be difficult and rarely patients are treated within this stipulated period for various reasons. ${ }^{13}$ Most of the cases are admitted and diagnosed at the time when this stipulated period is crossed. Hence it is unclear on to whether treating such patients of acute ischemic stroke with drugs other than thrombolytic agents would have any benefits in terms of reduction in severity of morbidity or improvement in functional ability. In fact we have planned a prospective study of large population size $(>200)$ to be conducted over a period of 4-5 years approved by institutional ethics committee to clear these doubts. However, prior to that study we planned to perform this retrospective pilot study to understand the drug utilization pattern and difficulties that might be faced while conducting the large and long duration prospective study. So the study aims at analysing the clinical and demographic factors associated with achieving improvement in functional ability by using Barthel Index (BI) score, an indicator of functional ability of post-stroke patients. ${ }^{14}$

\section{METHODS}

\section{Data source}

Study included cases of ischemic stroke admitted to a tertiary care teaching hospital of north karnataka, India over a period of one year (January 2015 to December 2015). After the approval of Institutional Ethics Committee, search for case records with stroke as diagnosis was conducted in the central medical records department of the hospital using the ICD-10 code I64. All the identified case records were further screened to select those cases with ischemic stroke. Cases admitted to our hospital after six hours but within seven days of onset of stroke with minimum of seven days of treatment after admission were considered for inclusion. The duration of follow-up and or analysis of outcome in our study were set to minimum of seven days of treatment because recovery and severity of morbidity at the end of seven days of treatment is supposed to indicate the outcome at 90 days of treatment. ${ }^{15}$ Cases were excluded if there is previous history of stroke, those associated with organic CNS disorders (tumours, cerebral venous thrombosis, cerebral infections, trauma) and if there is presence of other serious co-morbid illness which would interfere with recovery of the patient. Cases were confirmed of having ischemic stroke on the basis of MRI scan to rule out hemorrhagic stroke and other exclusion criteria.

\section{Data extraction}

From each case record data extraction and recording was done simultaneously by both the authors of the study in a pre-designed patient information sheet approved by Institutional Ethics Committee. All the relevant baseline demographic data were collected which included age, sex, time lapsed in initiating the treatment, duration of treatment, presence of hypertension and type-2 DM. Clinical data extracted included severity of stroke by modified National Institute of Health Stroke Scale (mNIHSS) score, daily blood pressure measurement, laboratory data on biochemical parameters like blood glucose, serum creatinine, serum sodium, serum potassium and plasma lipids levels. MRI scan of the brain provided the data with details on the location, area, side of infarction with type of stroke and artery affected. Drug utilization pattern of different classes of drugs used with the dose and duration of treatment of individual drugs was extracted. Patient was considered as treated with a drug provided the drug is administered for minimum of one day. Recovery of the patient from functional deficit at the end of treatment period was analysed by using BI score. Both mNIHSS score and BI score were not available in their original required format but were extrapolated by the author on the basis of the daily CNS examination findings and the performances of the patients recorded in the case records.

\section{Statistical methods}

The primary outcome measure used to quantify the functional recovery of the patient was BI score. Baseline demographic and clinical features and drug utilization pattern were described by descriptive statistics. Impact of various drugs, baseline and clinical factors on BI score was analysed by univariate analysis. Pearson's correlation was used for parametric variables with linear distribution and Spearman's correlation was used when two variables were nonparametric or parametric with non-linear distribution. However, considering that the BI score is non-parametric variable and the limited number of patients included in the analysis, we considered the values of spearman's correlation in reporting and analysing the strength of association. A subgroup analysis after exclusion of cases of posterior circulation infarction was planned to rule out the bias created due to lack of significant functional deficit observed in these category of patients. ${ }^{16}$ Similarly, a stratified subgroup analysis by stratifying cases according to severity of stroke was also conducted. Subgroup stratification of cases was done as cases with mNIHSS score $>15$ (moderate to severe stroke) and those with score $<15$ (minor stroke). ${ }^{17}$ These subgroup analyses were conducted only on those factors showing significant correlation with BI score. Statistical analysis was done by using SPSS software version 20 .

\section{RESULTS}

\section{Case record search}

Of the total 446 case records with diagnosis of stroke, only 39 met the eligibility criteria and included in the study. None of the cases were admitted and treated within the stipulated period of six hours and 227 case records were excluded because of duration of treatment being less 
than seven days. Of the total 446 cases, 219 cases treated for more than seven days were further screened for the eligibility. Of these 219 case records, except for 39 case records meeting eligibility criteria remaining 180 case records were excluded for various reasons like unavailability of MRI scan report $(n=32)$, diagnosed as case of hemorrhagic stroke $(n=64)$, being admitted after seven days of onset of stroke $(n=48)$, known old cases of stroke $(\mathrm{n}=16)$, complicated with other co-morbid conditions which may interfere with recovery of the patient $(n=20)$.

\section{Baseline features and drug utilization pattern}

Table 1 shows the baseline demographic and clinical features of the included patients along with their correlation with BI score.

Table 1: Baseline demographic and clinical factors and their correlation with BI score.

\begin{tabular}{|c|c|c|c|}
\hline \multirow{2}{*}{ Factors $(\mathbf{N}=39)$} & \multirow{2}{*}{ Mean \pm SD } & \multicolumn{2}{|c|}{ Spearman's correlation } \\
\hline & & R-value & P-value \\
\hline Age (years) & $57.6 \pm 14$ & -0.18228 & 0.26672 \\
\hline Sex ratio $(\mathrm{M}: \mathrm{F})$ & $17: 22$ & 0.21565 & 0.18734 \\
\hline Duration of treatment (days) & $9.72 \pm 4.03$ & -0.1706 & 0.29909 \\
\hline Time lapsed in treatment initiation (h) & $31.7 \pm 24.5$ & 0.41066 & $0.00941^{*}$ \\
\hline mNIHHS Score & $10.85 \pm 6.5$ & -0.51588 & $0.00078^{*}$ \\
\hline Presence of hypertension (n/N) & $25 / 39$ & -0.19853 & 0.22567 \\
\hline Presence of type-2 DM (n/N) & $12 / 39$ & 0.1719 & 0.29538 \\
\hline Both hypertension and type-2 DM & $10 / 39$ & -0.02261 & 0.90907 \\
\hline Day-1/Baseline SBP (mmHg) & $147.2 \pm 26.15$ & -0.39438 & $0.01298 *$ \\
\hline Day-1/Baseline DBP (mm Hg) & $91 \pm 16.2$ & -0.2547 & 0.11764 \\
\hline Final day SBP $(\mathrm{mmHg})$ & $131 \pm 12.7$ & 0.00158 & 0.99239 \\
\hline Final day DBP (mmHg) & $82.56 \pm 8.2$ & 0.13829 & 0.40116 \\
\hline Change in SBP (mmHg) & $-16.6 \pm 23.4$ & 0.42504 & $0.00699 *$ \\
\hline Change in DBP (mmHg) & $-8.46 \pm 15.8$ & 0.2828 & 0.08109 \\
\hline Baseline total cholesterol level (mg/dl) & $175 \pm 43.75$ & 0.05047 & 0.80659 \\
\hline Baseline triglyceride level (mg/dl) & $120.8 \pm 57.2$ & 0.32527 & 0.05888 \\
\hline Baseline LDL-cholesterol level (mg/dl) & $111 \pm 31.21$ & 0.15177 & 0.45923 \\
\hline Baseline HDL-cholesterol level (mg/dl) & $39.7 \pm 11.1$ & -0.16089 & 0.43237 \\
\hline Baseline hemoglobin $(\mathrm{g})(\mathrm{n}=35)$ & $13.15 \pm 2.55$ & 0.07658 & 0.66195 \\
\hline Baseline blood sugar level (mg/dl) & $127.36 \pm 42.3$ & -0.21985 & 0.51598 \\
\hline Baseline Serum creatinine level (mg) & $1.18 \pm 1.17$ & 0.36166 & $0.02568^{*}$ \\
\hline Baseline Serum sodium level (mEq/l) & $137 \pm 9.96$ & 0.24442 & 0.15706 \\
\hline Baseline Serum pottasium level (mEq/l) & $4.13 \pm 0.42$ & 0.03947 & 0.82189 \\
\hline
\end{tabular}

*Statistically significant $(\mathrm{P}=<0.05), \mathrm{N}=$ Total number of patients, $\mathrm{n} / \mathrm{N}$ for all plasma lipid parameters is $26 / 39, \mathrm{n} / \mathrm{N}$ for (random) blood sugar level is $11 / 39, \mathrm{n} / \mathrm{N}$ for baseline hemoglobulin is $35 / 39, \mathrm{n} / \mathrm{N}$ for serum sodium and potassium is $35 / 39$, $\mathrm{n} / \mathrm{N}$ for baseline hemoglobulin is $38 / 39$.

Of the 39 cases, 8 cases were not recognized with having any of these major co-morbid illnesses as past history. In the remaining 31 cases three were known cases of RHD with $\mathrm{AF}$ and remaining cases were having essential hypertension $(n=16)$ or type- 2 DM $(n=3)$ or both $(n=9)$. Only two cases records recorded the personal history of patients on alcohol consumption and tobacco smoking. Data on body weight of the patients was not available from any of the case records. Anterior and or middle cerebral arteries were involved in 29 of the total 39 cases. Right side and left side arteries were involved in 18 cases each and in remaining three cases there was infarction of bilateral arteries. No significant correlation was found between BI and involvement of the artery or side of infarct. Table 2 shows the major classes of drug used and their correlation with BI score. Drugs consistently prescribed in most of the patients included low dose aspirin, HMG-CoA inhibitors, amlodipine, mannitol, proton pump inhibitors, nootropic agents and vitamin Bcomplex tablets. Except for five patients who received $75 \mathrm{mg} / \mathrm{d}$ of low dose aspirin, remaining patients received $150 \mathrm{mg} / \mathrm{d}$. HMG-CoA inhibitors used were atorvastatin $(n=32)$ or rosuvastatin $(n=5)$ at the daily dose of $40 \mathrm{mg}$ or $10 \mathrm{mg}$ respectively. Amlodipine was the commonly prescribed anti-hypertensive in total 25 patients with hypertension. The dose of amlodipine used in 19 patients was $5 \mathrm{mg} / \mathrm{d}$ and in remaining patients it was at the dose of $10 \mathrm{mg}(\mathrm{n}=3)$ and $2.5 \mathrm{mg} / \mathrm{d}(\mathrm{n}=3)$. Injection mannitol 
$100 \mathrm{ml}$, i.v was used at varying frequency (BD or TID) for varying days (minimum one day to maximum ten days) in 30 patients. Three types of nootropic agents used in 19 patients were edavarone, piracetam and citicoline. Injection Edavarone at daily dose of $30 \mathrm{mg}$ was administered as slow i.v infusion. Piracetam was used orally at the dose of $800 \mathrm{mg}$ BD and citicoline at the dose of $500 \mathrm{mg}$ BD. Single therapy with edavarone $(n=5)$, piracetam $(n=2)$ was done in seven patients. Combined use of two type of nootropic agents as edavarone+piracetam $(n=3)$, edavarone+citicoline $(n=2)$ and piracetam+citicoline $(n=3)$ was done in most of these 19 patients. In four patients all three nootropic agents were used. Vitamin B-complex tablets used in 24 patients comprised mainly a combination of methycobalamine, pyridoxine and folic acid. Use of other drugs like beta-1 blockers $(n=3)$, insulin $(n=4)$, digoxin $(n=4)$, amiodarone $(n=2)$, intravenous fluids $(n=4)$, warfarin $(n=4)$, was limited to very few patients and hence were not considered as factors in estimating their correlation with BI score. In few numbers of patients, ACE-Is/ARBs $(n=8)$, diuretics $(n=6)$, antibiotics $(n=10)$, LMWH $(n=7)$ and oral glycerol $(n=8)$, oral anti-diabetic agents $(n=8)$ were used. Though the number was small, we still estimated their correlation with BI score and found insignificant association. In none of the cases thrombolytic agents were used. Physiotherapy as a modality of treatment was started within one day after admission in 29 of the 39 patients for a mean duration of 8 days $(8.7 \pm 4.2$ days). Depending on the type of neurological deformity the limb mobilization, gait training, standing exercises, etc were performed once daily regularly on daily basis.

Table 2: Major treatment modalities/drugs used and their correlation with BI score.

\begin{tabular}{|llll|}
\hline Factors & $\mathbf{n} / \mathbf{N}$ & Spearman's correlation \\
\hline & & R-value & P-value \\
\hline Low dose aspirin use & $37 / 39$ & -0.22937 & 0.16014 \\
\hline Statin use & $37 / 39$ & 0.06256 & 0.7052 \\
\hline Mannitol use & $30 / 37$ & -0.21014 & 0.19914 \\
\hline Oral glycerol use & $08 / 39$ & -0.03417 & 0.83639 \\
\hline LMWH use & $07 / 39$ & 0.10187 & 0.53717 \\
\hline Amlodipine use & $20 / 25$ & -0.0061 & 0.97743 \\
\hline ACEIs/ARBs use & $08 / 25$ & -0.3136 & 0.13564 \\
\hline Diuretic use & $06 / 39$ & -0.00637 & 0.96928 \\
\hline Beta-blocker use & $03 / 25$ & -0.18318 & 0.39157 \\
\hline OAD use & $08 / 12$ & 0.2531 & 0.42737 \\
\hline Nootropic agent use & $9 / 39$ & 0.10582 & 0.52143 \\
\hline B-complex use & $24 / 39$ & -0.26471 & 0.10342 \\
\hline Physiotherapy use & $29 / 39$ & -0.35813 & $0.02518^{*}$ \\
\hline
\end{tabular}

* Statistically significant $(\mathrm{p}=<0.05)$, OAD: Oral Anti-Diabetic drug

\section{Results of correlation}

Significant and negative correlation to BI score was seen with mNIHSS score, 'day-1 SBP' and 'use of physiotherapy' as factors. Significant positive correlation to BI score was associated with 'time lapsed in initiating the treatment', 'amount of decrease in SBP' and baseline 'serum creatinine level' as factors. None of the drug treatment had significant association with the BI score. Following a subgroup analysis after excluding patients of posterior circulation infarcts, significant correlations observed between BI score and 'serum creatinine level' was absent. On two subgroup analyses including cases with mNIHSS scores $>15$ and $<15$, following findings were observed. The significant and positive association of BI score with 'time lapsed in initiating the treatment' was absent in subgroup analysis including cases with mNIHSS score $>15$ and present in subgroup analysis including cases with mNIHSS score $<15$. With regard to negative association between BI score and 'use of physiotherapy' as treatment modality, there was lack of such significant association after two subgroup analyses including cases with mNIHSS score $>15$ and $<15$. There was lack of association between 'day-1 SBP' and BI score in subgroup analysis including cases with mNIHSS score $>15$ and present in subgroup analysis including cases with mNIHSS score <15. Similarly it was the same scenario with regard to 'change in SBP' as a factor. There was lack of significant association of baseline 'serum creatinine level' with BI score after two subgroup analyses including cases with mNIHSS score $>15$ and $<15$.

\section{DISCUSSION}

Since the scope of significant findings of any correlation statistics is limited to hypothesis generation rather than confirmation, here with we discuss the strength and direction of correlation between BI score and factors found to be significantly associated with it. Some of these associations are contradictory while some are justifiable according to the available evidences. The negative association between mNIHSS score, an indicator of severity of stroke and BI score and an indicator of functional recovery is self-explanatory. Perhaps this could be the reason why only the strength of correlation of mNIHSS score was found to be moderately strong (' $R$ ' value $=0.50$ to 0.70 ) while the remaining factors had weak (' $R$ ' value $=0.0$ to 0.30 ) correlation with BI score. ${ }^{18}$ In the methodological aspects of our study, mNIHSS score and BI score values were extracted after extrapolating the daily CNS examination findings mentioned in the case records. Perhaps the strength of correlation observed between these two may justify and indirectly indicate that the extrapolated mNIHSS score and BI score values are nearer to the real values.

Two factors found to have had persistent association with BI score after exclusion of cases of posterior circulation infarcts were 'time lapsed in initiating the treatment' and 'use of physiotherapy'. However, the positive association of achieving better functional ability by delayed initiation of treatment can be considering as spurious association. Since the basic concepts of starting a thrombolytic 
therapy within stipulated period of 4-6h of onset of stroke to improve the functional outcome in a patient of ischemic stroke is not applicable in these patients, there is a possibility of such spurious associations. Absence of positive association of BI score with 'time lapsed in initiating the treatment' in subgroup analysis including cases with mNIHSS score $>15$ and its presence in subgroup analysis including cases with mNIHSS score $<15$ perhaps indicate the reason for and presence of this spurious association. It appears that the mild nature of the severity of stroke rather than delayed initiation of treatment may be responsible for positive association with BI score observed in subgroup analysis including cases with mNIHSS score $<15$. On the other hand, for cases with severe stroke this factor of 'time lapsed in initiating the treatment' is an insignificant factor in determining the recovery of patient considering the severity of stroke and highlights the significance of initiation of thrombolytic therapy within stipulated time period. The lack of association between 'day-1 SBP' and BI score in subgroup analysis including cases with mNIHSS score $>15$ and its presence in subgroup including cases with mNIHSS score $<15$ can be explained on the similar grounds. Most of the patients with severe stroke usually present with very high baseline SBP due to severity of stroke and hence its negative correlation with BI score in subgroup analysis including cases with mNIHSS score $>15$. $^{19}$ Blood pressure level gradually decreases to near normal values after transient rise in initial days of acute stroke. ${ }^{19}$ Hence the scenario seen with regard to 'change in SBP' as a factor being positively associated with BI score indicates the pathophysiological process in these patients rather than the effect of blood pressure lowering agents. This could be the reason why there was no significant association between BI score and any of the anti-hypertensive drugs despite the presence of positive association of BI score with amount of 'change in SBP' achieved.

The negative correlation between BI score and 'use of physiotherapy' suggest negative effect of use of physiotherapy as a part of treatment modality on functional improvement. However there was lack of this significant association after subgroup analyses including cases with mNIHSS score $>15$ and $<15$. This may or may not indicate ineffectiveness of physiotherapy in these late admitted cases of stroke. The available evidences justify benefits of early use of physiotherapy as a treatment modality in better functional recovery of the stroke patients. ${ }^{20}$ Considering the influence of various factors like severity of stroke, time of initiation, duration, length and type of physiotherapy on its effectiveness, it is quite possible to have variable effects on the mobility of person and thus BI score observed in our study. ${ }^{20}$ Another possibility behind lack of any impact or association of 'use of physiotherapy' as factor with BI score in our study could be early assessment of the functional ability of the patients at the end of seven days. This could be too early to assess the effect of physiotherapy in these patients. Scenario of another spurious positive association found between baseline 'serum creatinine' level and BI score observed in our study indicates better functional recovery in patients with high serum creatinine level. This association is biologically not plausible and can be explained on the basis of absence of this association in two subgroup analyses of patients with mNIHSS score $>15$ and $<15$. All the spurious associations identified by subgroup analyses in our study highlight the influence of posterior circulation infarcts and mNIHSS score on functional recovery of the patients.

\section{CONCLUSION}

To conclude, there is a possibility of lack of any kind of benefits in terms of functional recovery achieved in late admitted cases of severe ischemic stroke at the end of seven days. Perhaps none of the treatment modality can have significant impact on this category of late admitted cases with moderate or severe stroke. Considering the lack of data on duration, length and type of physiotherapy used in these patients, the effect of physiotherapy cannot be confirmed. Mild cases of stroke also may not have the benefits of any treatment modalities considering the mild nature of the severity of stroke itself. Apart from the inherent drawbacks of the retrospective and uncontrolled design of our study, other major drawback of our study is the limitation on the availability of data. This is especially significant with regard to actual mNIHSS score and BI score values. Though retrospective extraction of mNIHSS score is found to correlate well with the actual mNIHSS score, validity of BI score is a major concern. ${ }^{21}$ Added to this, failure to identify other spurious associations and actual associations is quite possible here considering the small sample size of the study. Though we tried to identify the spurious associations by subgroup analyses, in fact the identification of such associations itself raises the question on validity of the other actual associations observed in our study. Considering these drawbacks and our idea behind conducting this pilot study, results of this study needs to be interpreted cautiously.

\section{Funding: No funding sources}

Conflict of interest: None declared

Ethical approval: The study was approved by the Institutional Ethics Committee

\section{REFERENCES}

1. Sims NR, Muyderman H. Mitochondria, oxidative metabolism and cell death in stroke. Biochimica et Biophysica Acta. 2009;1802:80-91.

2. Gupta R, Joshi P, Mohan V, Reddy S, Yusuf S. Epidemiology and causation of coronary heart disease and stroke in India. Heart. 2008;94:16-26.

3. Edward CJ, Brian S. Ischemic Stroke Medication. Medscape: drugs and diseases, 2015. Available at http://emedicine.medscape.com/article/1916852medication\#showall. Accessed 28 December 2015. 
4. Edward CJ, Brian S. Ischemic Stroke Treatment \& Management. Medscape drugs and diseases. 2015. http://emedicine.medscape.com/article/196852treatment\#aw2aab6b6b4. Accesses 28 December 2015.

5. Howard SK, José B, Alfred SC. Long-Term Therapy to Prevent Stroke. J Am Board Fam Med. 2005;18:528-40.

6. Wang H, Tang Y, Rong X, Li H, Pan R. Effects of Early Blood Pressure Lowering on Early and LongTerm Outcomes after Acute Stroke: An Updated Meta-Analysis. PLoS ONE. 2014;9:e97917.

7. Jeffery LS. Targeting the Brain: Neuroprotection and Neurorestoration in Ischemic Stroke Pharmacotherapy. 2010;30:S62-9.

8. Kiyoshi K, Hisaaki U, Motohiro M, Yoshinaka M, Elichiro T. Clinical Neuroprotective Drugs for Treatment and Prevention of Stroke. Int. J. Mol. Sci. 2012;13:7739-61.

9. Helmi LL, Wayne MC. Neuroprotective Agents in Stroke- Overview of Neuroprotective Agents. Medscape drugs and diseases. 2015. Available at http://emedicine.medscape.com/article/1161422overview\#a30. Accessed 28 December 2015.

10. Diener HC. Primary and secondary stroke prevention with antiplatelet drugs. Curr Pharm Des. 2006;12:1293-7.

11. Lian Z, Stan H, Philip MW. Antiplatelet Therapy for Stroke Prevention. Br J Cardiology. 2005;12:57-60.

12. Powers WJ, Derdeyn CP, Biller J, Coffey CS, Hoh BL, Jauch EC. American Heart Association/American Stroke Association Focused Update of the 2013 Guidelines for the Early Management of Patients With Acute Ischemic Stroke Regarding Endovascular Treatment: A Guideline for Healthcare Professionals From the American Heart Association/American Stroke Association. Stroke. 2015;46:3020-35.

13. Sankapithilu GB, Nagaraj V, Khan MA. A study of factors delaying hospital arrival and predictors of mortality in patients presenting to emergency department with Stroke: A developing state scenario. Online Journal of Health and Allied Sciences. 2010;9:1-4.

14. Sulter G, Steen C, De Keyser J. Use of the Barthel index and modified Rankin scale in acute stroke trials. Stroke. 1999;30:1538-41.

15. Ovbiagele B, Saver JL. Day-90 acute ischemic stroke outcomes can be derived from early functional activity level. Cerebrovasc Dis. 2010;29:50-6.

16. Helseth EK. Posterior Cerebral Artery Stroke. 2015. Available http://emedicine.medscape.com/article/2128100overview\#a7. Accessed 30 December 2015.

17. National Institutes of Health Stroke Scale, 2015. Available at https://en.wikipedia.org/ wiki/National_Institutes_of_Health_Stroke_Scale. Accessed 30 December 2015.

18. Mukaka MM. Statistics corner: A guide to appropriate use of correlation coefficient in medical research. Malwi medical Journal. 2012;24(3):69-71.

19. Qureshi AI. Acute hypertensive response in patients with stroke: pathophysiology and management. Circulation. 2008;118:176-87.

20. Pollock A, Baer G, Campbell P, Choo PL, Forster A, Morris J, et al. Physical rehabilitation approaches for the recovery of function and mobility following stroke. Cochrane Database of Systematic Reviews 2014;4:CD001920.

21. Kasner SE, Cucchiara BL, McGarvey ML, Luciano JM, Liebeskind DS, Chalela JA. Modified National Institutes of Health Stroke Scale Can Be Estimated From Medical Records. Stroke. 2003;34:568-70.

Cite this article as: Hiremath SB, Sudhindra PA. A retrospective pilot study analyzing the effects of various factors and benefits of various drugs used in treating late admitted cases of acute ischemic stroke. Int J Basic Clin Pharmacol 2016;5:502-7. 